\title{
Long term effect of hypermetropic anisometropia on the visual acuity of treated amblyopic eyes
}

\author{
Shmuel Levartovsky, Moshe Oliver, Nava Gottesman, Michael Shimshoni
}

\begin{abstract}
Aim-To evaluate the effect of the extent of hypermetropic anisometropia on the long term visual acuity results in amblyopic eyes following their treatment by occlusion.

Methods-The visual acuity of 86 patients, who had been treated for unilateral amblyopia by occlusion of the fellow eye and followed up at least to the age of 9 years, was examined 6.4 years, on average, after cessation of treatment. Patients were divided into two groups-those with a small amount of hypermetropic anisometropia, where the spherical equivalent difference between the eyes ranged between 0 and +1.50 dioptres, and those with a large amount of hypermetropic anisometropia, where the difference was +1.75 dioptres or greater.

Results-Deterioration of visual acuity after cessation of occlusion treatment occurred in $51 \%$ of the patients in the group with a small amount of anisometropia and in $75 \%$ of the patients in the group with a large amount. At cessation of treatment, the average visual acuity in both groups was $20 / 40+$. At the long term follow up examination, however, the average visual acuity was 20/40and $20 / 70$, respectively. This difference was statistically significant.

Conclusions-Hypermetropic anisometropia greater than 1.50 dioptres appears to be a risk factor for deterioration of visual acuity in the long term, following treatment of amblyopic eyes by occlusion of the fellow eye.

(Br f Ophthalmol 1998;82:55-58)
\end{abstract}

Occlusion of the fellow eye is the most widely accepted and probably the most effective method of treatment for unilateral amblyopia. Upon cessation of treatment, however, the improved visual acuity in the amblyopic eye tends to deteriorate. In a previous study, ${ }^{1}$ we showed that deterioration of visual acuity had occurred in $55 \%$ of treated eyes when examined at a long term follow up examination. The age at which treatment was started did not significantly affect the extent of deterioration or the mean visual acuity in the long term. In another study ${ }^{2}$ we found that low initial visual acuity in the amblyopic eye before treatment and strabismic anisometropic amblyopia are risk factors for deterioration of visual acuity in the long term. In the present study we considered an additional variable, the amount of hypermetropic anisometropia, as a possible risk factor and examined its long term effect on the deterioration of visual acuity in eyes that had earlier been treated for amblyopia.

\section{Patients and methods}

Between 1974 and 1979, 350 children with unilateral amblyopia were diagnosed and treated in the outpatient eye clinic of Kaplan Medical Centre, Rehovot. Our report of that series ${ }^{3}$ contains a detailed description of the patients and methods, which is also applicable to the present work. Treatment consisted only of the use of spectacles and occlusion of the fellow eye. In each case, after the best visual acuity in the amblyopic eye was attained, we continued to monitor vision every 3 months for a year. Thereafter, visual acuity was checked during the course of a complete eye examination performed at least once a year until the child was 9 years old. If the visual acuity had deteriorated occlusion of the fellow eye was reinstated, usually for a few weeks, in order to restore vision to the level previously attained.

Inclusion of a patient in the present long term follow up study was contingent on the following criteria being met at the time that amblyopia was diagnosed and during the subsequent period of treatment and monitoring up to the age of 9 :

(1) Compliance with an age suitable Snellen chart for the initial visual acuity examination. An exception was made for 12 children with unilateral tropia who were too young (between 2 and 3 years of age) at the beginning of the study to be tested in this way. We estimated the visual acuity of the amblyopic eye in these children by determining the density of the filter required to induce a shift of fixation to the squinting or presumed amblyopic eye. Only two children of whom the initial visual acuity was determined by the above technique fulfilled all the criteria and were included in the present study.

(2) An initial visual acuity of $20 / 60$ or worse in the amblyopic eye.

(3) Full compliance with the instructions of the medical staff during the period of therapy and follow up.

(4) Cessation of occlusion treatment for at least a year before the present study.

On the basis of these criteria 157 children were eligible for inclusion in the present study, and $104(66 \%)$ of them responded to our invitation and presented themselves for the long term follow up examination. Of these 104 children, 94 had been monitored for visual acuity up to at least the age of 9 years. Eight of them had myopic anisometropia and were excluded from the study because of the small sample 
Table 1 Number (\%) of patients who showed deterioration in visual acuity (VA) at the long term follow up examination

\begin{tabular}{ll}
\hline Anisometropia & $\begin{array}{l}\text { No (\%) of patients who showed } \\
\text { deterioration in VA }\end{array}$ \\
\hline$\leqslant 1.50 \mathrm{D}(\mathrm{n}=74)$ & $38(51)$ \\
$\geqslant 1.75 \mathrm{D}(\mathrm{n}=12)$ & $9(75)$ \\
\hline
\end{tabular}

size. The study population, therefore, consisted of 86 patients. At the long term follow up examination, the best corrected visual acuity on the Snellen chart was recorded. When necessary, cycloplegic refraction was performed 1 hour after administration of cyclopentolate $1 \%$, twice during a 10 minute interval, followed by subjective refraction a week later. Orthoptic and fundal examinations were also performed.

According to the difference in cycloplegic refraction between the two eyes at the child's initial examination, two groups of patients were defined:

(1) small amount of anisometropia; in 74 patients the difference between the spherical equivalents of the amblyopic eye and the good eye was less than or equal to +1.50 dioptres.

(2) large amount of anisometropia; in 12 patients the difference between the spherical equivalents was +1.75 dioptres or greater.

DATA ANALYSIS AND CALCULATION OF VISUAL ACUITY DETERIORATION

Following Moseley and Jones ${ }^{4}$ we converted each visual acuity score on the Snellen chart to its logarithmic value, termed the $\log$ MAR value. Means and standard errors of each group were computed using the $\log M A R$ values, as were the statistical significance levels of visual acuity differences between the groups. For brevity, we will refer to these $\log M A R$ values as "visual acuity scores", except where otherwise stated.

The visual acuity score at the long term follow up examination was compared with that attained by the child upon termination of occlusion treatment. The difference between the two was the "deterioration score", which

Table 2 Mean visual acuity scores (SE) in $\log M A R$ values and their equivalent Snellen chart values at the beginning and at the end of occlusion therapy and at the long term follow up examination

\begin{tabular}{|c|c|c|c|c|c|c|}
\hline \multirow[b]{2}{*}{ Anisometropia } & \multicolumn{2}{|c|}{$\begin{array}{l}\text { VA at commencement of } \\
\text { occlusion treatment }\end{array}$} & \multicolumn{2}{|c|}{$\begin{array}{l}\text { VA at termination of } \\
\text { occlusion treatment }\end{array}$} & \multicolumn{2}{|c|}{$\begin{array}{l}\text { VA at long term follow } \\
\text { up }\end{array}$} \\
\hline & $\log M A R$ & Snellen & $\log M A R$ & Snellen & $\log M A R$ & Snellen \\
\hline$\leqslant 1.50 \mathrm{D}$ & $0.89(0.05)$ & $20 / 155$ & $0.26(0.02)$ & $20 / 36$ & $0.34(0.02)$ & $20 / 44$ \\
\hline$\geqslant 1.75 \mathrm{D}$ & $0.87(0.09)$ & $20 / 149$ & $0.26(0.03)$ & $20 / 36$ & $0.55(0.10)$ & $20 / 70$ \\
\hline
\end{tabular}

$\mathrm{VA}=$ visual acuity; $\log \mathrm{MAR}=$ logarithm of the minimum angle of resolution

Table 3 Average amount of visual acuity deterioration * (SE) and average amount of overall improvement ${ }^{*}$ in visual acuity (SE) in the two groups at the long term follow up examination in $\log M A R$ values

\begin{tabular}{lll}
\hline Anisometropia & $\begin{array}{l}\text { Average deterioration in visual } \\
\text { acuity }(S E)\end{array}$ & $\begin{array}{l}\text { Average overall improvement in } \\
\text { visual acuity }(S E)\end{array}$ \\
\hline$\leqslant 1.50 \mathrm{D}$ & $-0.08(0.02)$ & $0.55(0.04)$ \\
$\geqslant 1.75 \mathrm{D}$ & $-0.28(0.10)$ & $0.33(0.14)$ \\
& $\mathrm{p}<0.021$ & $\mathrm{p}<0.031$
\end{tabular}

$\star$ Deterioration is defined as the difference between the visual acuity score at the long term follow up examination and at the end of treatment, and overall improvement is defined as the difference between the visual acuity score at the long term follow up examination and at the commencement of treatment. reflects the amount of deterioration in visual acuity. For each of the two groups defined above, the average deterioration was calculated by dividing the sum of the deterioration scores by the number of children in the group. In addition, each patient's "improvement score" was defined as the difference between the visual acuity score at the long term follow up examination and at the commencement of treatment. It should be stated that an improvement or deterioration of $0.3 \log M A R$ is a halving or doubling of the visual angle that can be resolved.

The data for each child, including visual acuity before occlusion treatment was started, refraction of both eyes, cover test findings at the commencement of treatment as well as best visual acuity at termination of treatment, and best visual acuity at the long term follow up examination, were prepared for analysis. The Student's $t$ test and the $\chi^{2}$ test were used for statistical evaluation. Results were assumed to be significant if $\mathrm{p}<0.05$.

\section{Results}

Of the 86 patients included in this study, 41 were males and 45 were females. Their ages at the initial examination ranged from 2 to 11.5 years (in $80 \%$ of the patients from 3 to 8 years). Their ages at the present long term follow up examination ranged from 9.0 to 20.0 years (mean 13.7 (SD 2.5)). The latter examination was performed between 1.1 and 9.8 years (average $6.4(1.9)$ ) after discontinuation of occlusion treatment. Most of the children included in the study $(84 \%)$ were examined between 4 and 9 years after the treatment was discontinued. In 70 children $(81 \%)$ strabismus was found in the initial examination, while 16 children (19\%) were orthophoric.

Thirty nine $(45 \%)$ of the examinees were found to have maintained the visual acuity they had achieved when occlusion treatment was terminated, while in $47(55 \%)$ the visual acuity had deteriorated.

AMOUNT OF ANISOMETROPIA AND LONG TERM VISUAL ACUITY DETERIORATION

Deterioration in visual acuity was seen in both groups at the long term follow up examination (Table 1). The difference between the two groups was not statistically significant.

Table 2 presents the mean visual scores in logarithmic values as well as in Snellen chart values obtained by the two groups at the beginning and end of treatment and at the long term follow up examination.

Table 3 presents the average amount of deterioration and of overall improvement (defined as in Methods) in visual acuity in the two groups, expressed as logMAR values. Although the two groups had similar visual acuity scores at the beginning and at the end of treatment, the amount of deterioration in visual acuity at the long term examination was significantly higher in the group with a large amount of anisometropia $(\mathrm{p}<0.05)$. The overall improvement was significantly better in the group with a small amount of anisometropia $(p<0.05)$. 


\section{Discussion}

In our previous reports ${ }^{12}$ on patients from the same study group as the present one, the long term results of occlusion treatment for amblyopia were analysed 6.4 years, on average, after treatment was discontinued. We found that visual acuity attained at the end of occlusion treatment was maintained in $47 \%$ of the treated amblyopic eyes. This finding concurs with the results of other investigators, ${ }^{5-11}$ who reported that $10 \%$ to $44 \%$ of treated eyes maintained their visual acuity at examinations performed 1 to 10 years after termination of occlusion treatment. We assume that the higher success rate in our case can be explained by the fact that we restricted participation in the study to those children who had complied fully with the instructions of the medical staff during the period of therapy and monitoring and were followed up to at least the age of 9 years.

The age at which therapy was initiated did not significantly affect the visual acuity results or the amount of deterioration of visual acuity measured at the long term follow up examination. ${ }^{1}$ However, low initial visual acuity and strabismic-anisometropic amblyopia were found to be significantly associated with greater deterioration of vision at the long term follow up examination of treated eyes. ${ }^{2}$

In the present study we evaluated the extent to which the amount of hypermetropic anisometropia affects the stability of visual acuity attained at the end of occlusion treatment, measured 6.4 years on average after termination of the therapy.

Long term visual acuity outcome was compared in two groups of patients with unilateral amblyopia - a group with a small amount of anisometropia where the difference in spherical equivalent between the two eyes ranged from 0 to +1.50 dioptres and a group with a large amount of anisometropia, where the difference between the eyes was +1.75 dioptres or greater. The determination of the amount of anisometropia for each group was based mainly on our clinical experience which bears out that larger amounts of anisometropia have a worse prognosis for successful treatment. More meaningful results could be obtained by analysing subgroups with increments of 0.50 dioptres. However, statistical analysis in this case would be meaningless. We studied consecutive cases of amblyopia presenting to our paediatric strabismus clinic and therefore the naturally occurring prevalence of the varying severity of anisometropic hypermetropia in children with amblyopia is reflected in our study (the low anisometropia group has six times as many patients as the high group). Moreover, the subdivision used in this study enabled a comparison of the long term visual acuity results of treated amblyopic eyes with low versus high anisometropia. Our findings showed that the visual acuity results at the long term follow up examination of the latter group compared unfavourably with those of the former. Although the two groups had achieved equal visual acuity results (average $20 / 40+$ ) at the end of occlusion treatment, at the long term follow up examination the group with a larger amount of anisometropia showed significantly greater deterioration in vision (average visual acuity of 20/70 compared with $20 / 40$ in the group with a small amount of anisometropia). Treatment of anisometropic amblyopia has a high success rate. Flynn and Cassady reported a success rate of almost $80 \%$ in achieving visual acuity of $20 / 40$ in amblyopic eyes with strabismic anisometropia or anisometropia. ${ }^{11}$ Sen reported improvement of at least two lines on the Snellen chart in $63.7 \%$ of patients treated for anisometropic amblyopia. ${ }^{12}$ However, the long term stability of the visual improvement was not addressed in either of those studies.

Kutschke et al ${ }^{13}$ treated 124 patients with anisometropic amblyopia. In that study the 42 patients with hypermetropic anisometropia had a mean initial visual acuity of $20 / 136$, and their best visual acuity at the end of treatment was $20 / 26$. In 16 patients with compound hypermetropic astigmatism, the mean initial visual acuity was $20 / 187$ and the best visual acuity achieved at the end of therapy was $20 / 33$. The above study population can be compared with that of the present study, as we had included patients with hyperopia and compound hyperopic astigmatism. In our series, the initial visual acuity for the whole group was 20/150 and the best visual acuity achieved at the end of occlusion therapy was $20 / 40+$ on average. Kutschke et al reported that of 66 who had completed the prescribed occlusion treatment, $16(24.2 \%)$ deteriorated by two or more lines of visual acuity after occlusion treatment was discontinued. The length of the follow up period after cessation of occlusion treatment was not mentioned. In our study, $55 \%$ of patients in both groups showed deterioration in visual acuity; these results were recorded 6.4 years, on average, after all occlusion treatment was discontinued.

Woodruff et $a l^{14}$ analysed data on 961 patients who were treated for amblyopia by occlusion treatment at seven orthoptic centres. The visual acuity value used for analysis of the outcome of therapy in each patient was the best visual acuity recorded within three visits of treatment termination. In children with anisometropia, the final visual acuity was found to be significantly related to the difference in spherical equivalent between the two eyes. The worst final visual acuities were associated with the highest degrees of anisometropia.

We conclude that a large amount of hypermetropic anisometropia-that is, a difference of more than +1.50 dioptres in spherical equivalent between the amblyopic and the fellow eye, has an unfavourable effect on the long term maintenance of visual acuity after discontinuation of occlusive treatment. This raises the question whether monitoring and treatment should be continued until a later age in children with hypermetropic anisometropia and amblyopia higher than 1.50 dioptres. Further studies are needed to clarify this assumption. 
1 Levartovsky S, Gottesman N, Shimshoni M, Oliver M. Factors affecting long-term results of successfully treated amblyopia: age at beginning of treatment and age at cessation of monit

2 Levartovsky S, Oliver M, Gottesman N, Shimshoni M. Factors affecting long-term results of successfully treated amblyopia: initial visual acuity and type of amblyopia. $\mathrm{Br} f$ Ophthalmol 1995; 79:225-8.

3 Oliver M, Neumann R, Chaimovitch Y, Gotesman N, Shimshoni $M$. Compliance and results of treatment for amblyopia in children more than age 8 old. Am f Ophthalmol 1986;102:340-5.

4 Moseley MJ, Jones HS. Visual acuity: calculating appropriate averages. Acta Ophthalmol Copenh 1993;71:296-300.

5 Sparrow JC, Flynn JT. Amblyopia: a long-term follow-up. $\mathcal{F}$ Pediatr Ophthalmol 1977;14:333-6.

6 Gregersen E, Rindziunsky OT. "Conventional" occlusion in the treatment of squint amblyopia. A 10-year follow-up. Acta Ophthalmol (Copenh) 1965;43:462-74.
7 Malik SRK, Virdi PS, Goel BK. Follow-up results of occlusion and pleoptic treatment. Acta Ophthalmol (Copenh)

8 Aichmair H, Frey RG. Late results from amblopia treatment. Klin Monatsbl Augenheilkd 1968;153:214-8.

9 Schropfer HD, Meinert K. Late results in amblyopia treatment. Klin Monatsbl Augenheilkd 1972;166:315-20.

0 Leydhecker W, Ricklefs G, Ruhling R. Late results of amblyopia treatment. Klin Monatsbl Augenheilkd 1967;151: 373-6.

11 Flynn JT, Cassady JC. Current trends in amblyopia therapy. Trans Am Acad Ophthalmol Otolaryngol 1978;85:428-50.

12 Sen DK. Results of treatment of anisohypermetropic amblyopia without strabismus. Br f Ophthalmol 1982;66: $680-4$

13 Kutschke PJ, Scott WE, Keech RV. Anisometropic amblyopia. Ophthalmology 1991;98:258-63.

14 Woodruff G, Hiscox F, Thompsom JR, Smith LK. Factors affecting the outcome of children treated for amblyopia. affecting the outcom
Eye $1994 ; 8: 627-31$ 